\section{Quantum Conduction: a Step-by-Step Guide}

\author{
Frederic 0tt \\ James Lunney \\ Trinity College, Dublin
}

When you open an electrical contact, the junction resistance changes from near zero to infinity in a very short time. But what happens in between? Many possible scenarios come to mind: stretching of a neck of metal to form a nanowire, ballistic electron transport and electron tunnelling, to name but a few.

A measurement is called for. Here we show that the measurement can be very easy and the result dramatic: the conduction is quantised.

\section{A Short History}

In 1987, Gimzewski and Moller ${ }^{1}$ observed that when the tip of a scanning tunnelling microscope (STM) was brought into contact with a silver substrate the contact resistance suddenly decreased to a few $\mathrm{k} \Omega$. This was the first hint of quantum conductance (QC) in nanowires. However, when van Wees et $a l^{2}$ and Wharam et $a l^{3}$ independently reported their measurements of conductance quantised in units of $G_{0}=2 e^{2} / h$ in $2-D$ electron-gas point contacts, it came as a surprise, even though there had been extensive theoretical discussion on the resistance of ballistic wires.
A few years later experiments by Pascual et al ${ }^{4}$ showed clear evidence of quantisation of the conductance in the metallic 'nanowires' formed when an STM tip was pulled away from a sample. At about the same time the mechanically controllable break junction technique was developed. Here, a thin wire was broken by bending the substrate on which it was glued. Very recently, Costa-Kramer et al have shown that the effect could be observed simply by pulling apart two macroscopic wires in electrical contact. Now, ten years after the discovery of QC, students can set up a very simple experiment to observe quantised conduction. The experiment uses an electromechanical relay switch to break the contact and works at ambient pressure and room temperature.

\section{A Student-Proof Experiment}

It is easy to build a simple and cheap setup to observe quantised conduction. The main components are shown in figure 1. We have used contacts in simple electromechanical relays which work surprisingly well. Commercial relays have different

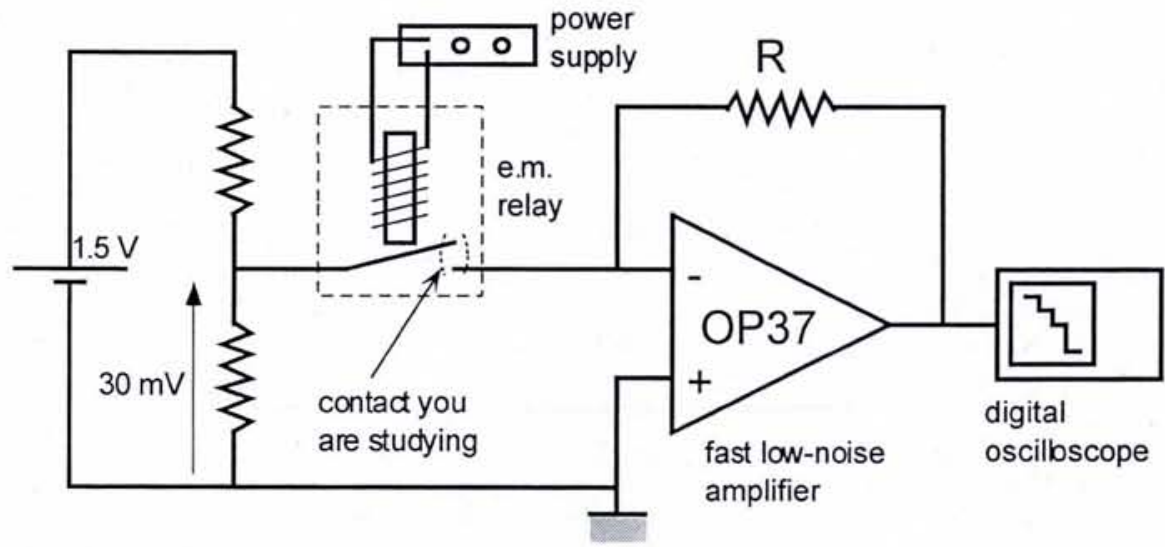

Fig 1 The set-up used to observe quantum conductance

types of contact coating; noble metals ( $\mathrm{Au}$ alloys or AgCdO) work well for quantum conduction.

The experiment is performed by opening the contact of the e.m. relay while recording the resistance of the contact. Typically, the contact is biased with a few tens of $\mathrm{mV}$. The current is measured using an $I / V$ converter based on a fast low noise amplifier (OP37 for example). The amplifier output is directly connected to a digital oscilloscope. The measured conductance $\mathrm{G}$ of the contact is proportional to the output voltage and equal to:

$$
G=1 / R_{1}=-V_{\text {output }} / R_{2} V_{\text {bias }}
$$

\section{First Quantum Steps}

Once you've spent a couple of hours wiring the small amplifier, getting a record of quantum conduction only takes a few minutes. Figure 4 shows typical traces. One of those is exceptional, figure 4(a), showing five well defined plateaux corresponding to integer values of $\mathrm{G}_{\mathrm{o}}$. However, in general you may get less well defined steps, of the kind shown in figure $4(b)$. Usually, the low order steps near $G_{0}$ or $2 G_{0}$ are well defined but the individual traces vary widely from one to the other, and non-integer steps are often observed. This is not a problem specific to this set-up; even in elaborate STM experiments, where

\section{Small but Perfectly Fast}

Researchers at Bell Labs in the US have built a nanotransistor for the next millennium. Just 182 atoms wide, it's four times thinner than the smallest transistors used today. And it's a complete semiconductor transistor: 60 nanometers of source, drain and metal oxide gate, all of which is very much in the realms of the quantum, writes Toby Chapman.

'There's a lot of tunnelling current that goes through these very thin oxides,' says Steven Hilenius, Head of Bell's silicon device research department.'One of the major problems with this is to plan in the circuits on having current going through the gates.'

It's not the first nanotransistor, but it's a practical design, forecast to go into service in the year 2009. And anyway, it was announced to coincide with last December's fiftieth anniversary of the invention of the transistor.

And the reason that we have spent fifty years trying to make them as small as possible, according to Hilenius, is so that we can make as dense a circuit as possible. The new device does the same job as today's devices, but for around a hundredth of the power. 
the breaking speed is accurately controlled, results are generally not reproducible. There is as yet no method to break a contact in a reproducible way. This has prompted some workers to accumulate the conductance traces from a large number of breaks and plot a histogram of the average behaviour of breaking contacts. The averaged conductance of a nanowire usually shows well defined low order steps (2 to 3 ) with an increasing spreading of the quantisation step as the order increases. Above the third order, no clear quantisation has been observed on average, although it may appear in some individual events. Several explanations have been put forward: disorder, impurities and surface roughness among them.

A simple follow-up to the experiment can investigate what happens with other materials brought into electrical contact (simply mount the samples on the relay arms). Gold works well, whereas transition metals usually give poor results. It has been shown by Agrait $e t$ al that the steps in conductance are always correlated with major structural reconstructions in the nanowire, and thus depend on the mechanical properties of the materials. Correia $e t$ al $^{8}$ have also been able to directly look at what is happening at the contact using electron microscopy. They show that the contact breaking proceeds as in figure 6: a large neck is stretched into many nanofilaments with a fractal structure. Again, this mechanism is materialdependent.

\section{Yet to be Explored}

Many aspects of such point contacts remain to be explored. These include localisation, magnetotransport (magnetic switching and magnetic blockade), dynamic effects and nonlinear effects (those related to the conduction current, for example). Possible applications such as atomic-sized switches have been suggested. Figure 5 shows oscillatory behaviour when the contact is poised near the edge of a step, which is thought to be related to mechanical instabilities.

The effect of QC in metallic nanowires is still not fully understood. Recent observation and calculation has shown that the origin of the quantised conductance may be more complex than suggested by the simple adiabatic theory. The measurement by $\mathrm{E}$. Sheer ${ }^{9}$ in superconducting $\mathrm{Al}$ has shown that even the steps near the first quantum of conductance can arise from the contributions of up to 4 quantum modes. Similar effects have also come to
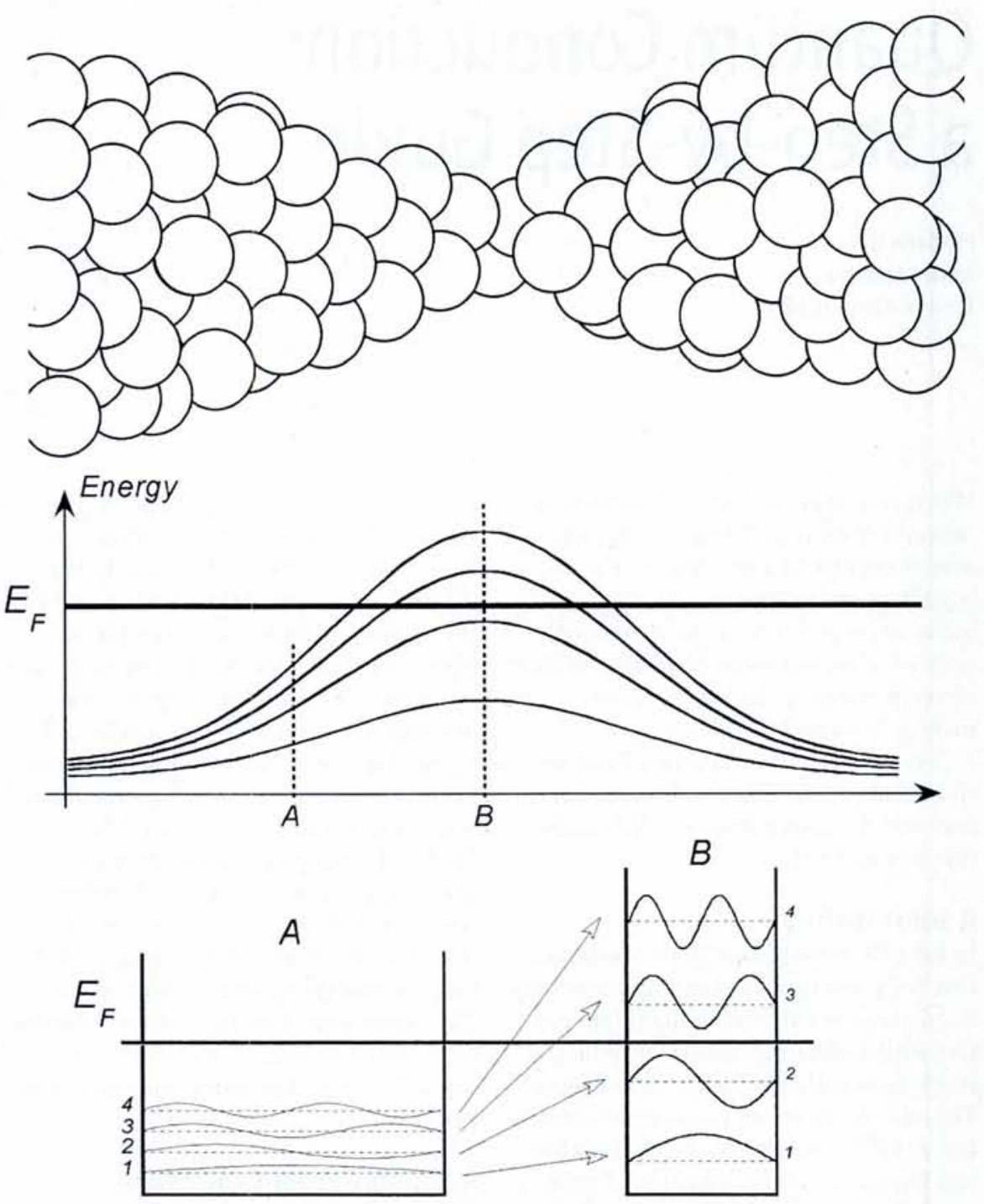

Fig $2 \mathrm{a}$ Above The electrons flowing through the nanowire have to overcome a potential barrier. The Fermi level determines the number of transverse modes which carry the current

Fig $\mathbf{2 b}$ Below Band structure in a perfect nanowire. The splitting between the channels can be very large

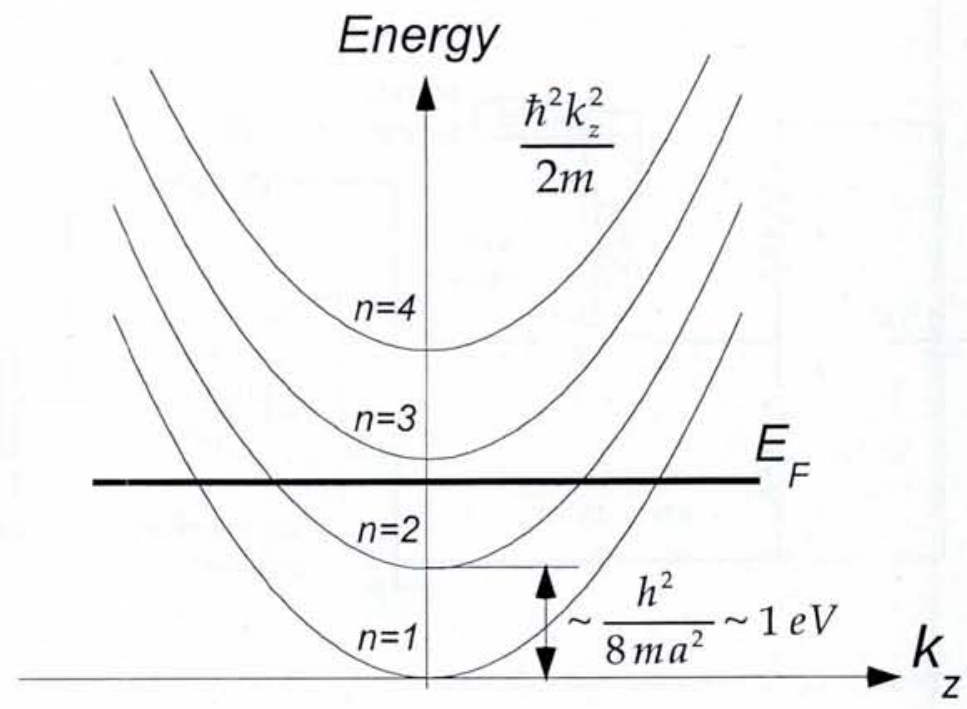


Fig 3 A nanowire connecting two electron reservoirs

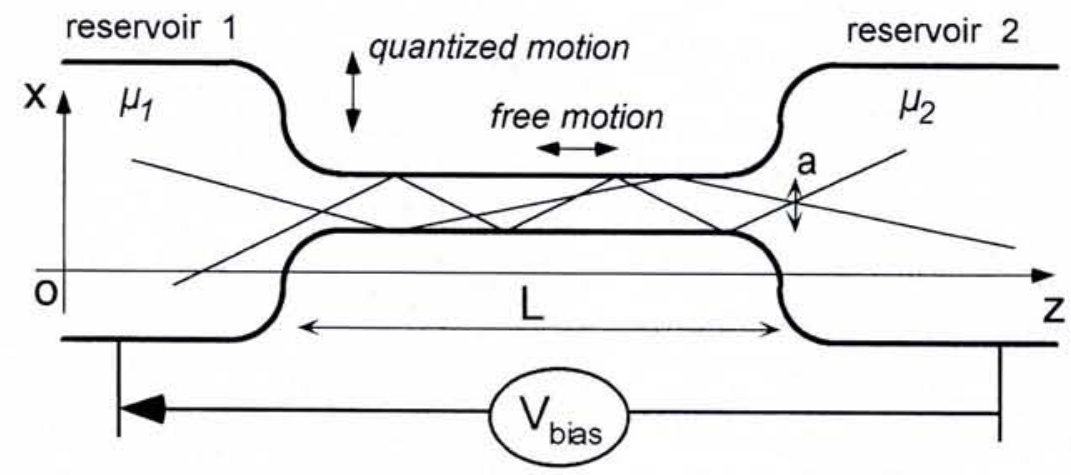

Fig 4 Some traces observed when opening a relay contact: (a) an exceptional trace showing 5 levels of quantisation, (b) a more typical trace
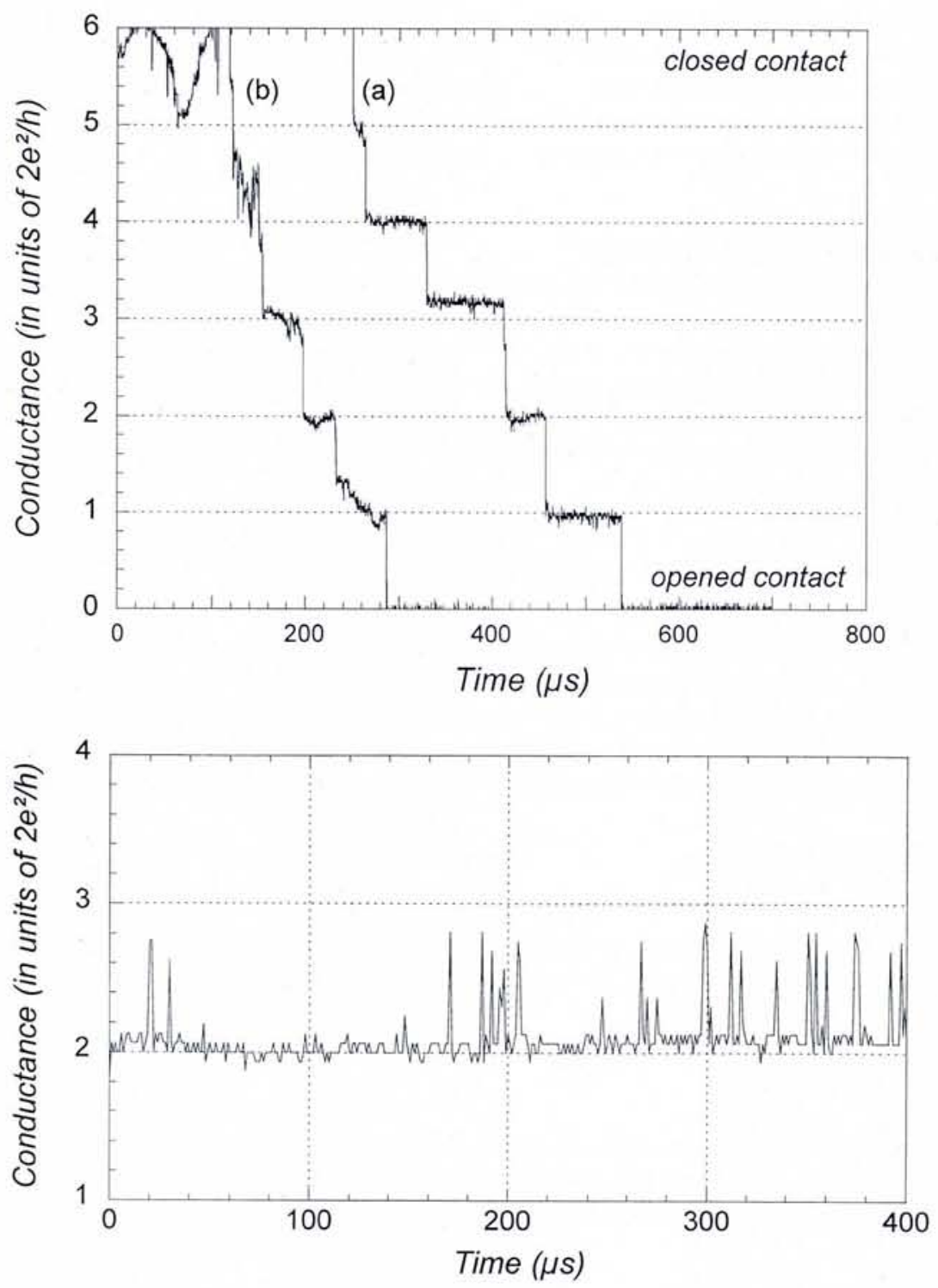

Fig 5 Noise in the conductance arising from mechanical instabilities between two configurations of the nanowire

( $\mu s)$

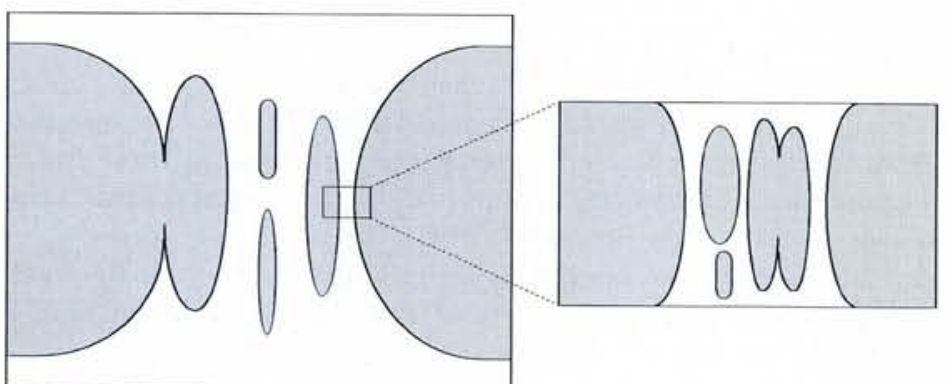


light in theoretical calculations ${ }^{10}$. These results suggest that even though the simple theory is in good agreement with some experiments, there is still much work to be done.

The field of ballistic transport in nanostructures has also triggered studies in other fields. It is surprising that even though single slit optical diffraction was first described in the 19th century, the optical counterpart of quantised conduction was observed as late as $1991^{11}$ : the light transmitted through a slit of increasing width is also quantised. The next step is to observe this type of quantisation for the supercurrent in superconductors.

Quantised conduction was observed in high-tech labs ten years ago and can now be seen in a simple table-top experiment. Will it spread even further and appear in electronic devices in the future? The science of nanostructures holds this promise. The future may indeed be paved with quantum steps.

\section{References}

[1] J.K. Gimzewski, R. Moller, Phys. Rev. B 36, 1284 (1987).

[2] B. J. van Wees, H. van Houten,

C.W.J. Beenakker, J.G. Williamson,

L.P. Kouwenhoven, D. van der Marel, C.T. Foxon, Phys. Rev. Lett. 6o, 848 (1988).

[3] D.A. Wharam, T. J. Thornton, R. Newbury, M. Pepper, H. Ahmed, J.E.F. Frost, D.G. Hasko, D.C. Peacock, D.A. Ritchie, G.A.C. Jones, J. Phys. C 21, 209 (1988).
[4] J.I. Pascual, J. Mendez, J. Gomez-Herrero, A.M. Baro, N. Garcia, Vu Thien Binh, Phys. Rev. Lett. 71, 1852 (1993).

[5] J.L. Costa-Krämer, N. Garcia, P. GarciaMochales, P.A. Serena, Surface Science 342, 1144 (1995).

[6] R. Landauer, IBM J. Res. Dev. 1, 223 (1957). [7] N. Agrait, G. Rubio and S. Vieira, Phys. Rev. Lett. 74, 3995 (1995).

[8] A. Correia, M.I. Marques and N. Garcia, J. Vac. Sci. Technol B 15, 548 (1997).

[9] E. Sheer, P. Joyez, D. Esteve, C. Urbina, M.H. Devoret, Phys. Rev. Lett. 78, 3535 (1997).

[10] P. Garcia-Mochales, P.A. Serena, Phys. Rev.

Lett. 79, 2316 (1997).

[11] E.A. Montie, E.C. Cosman, G.W.t Hooft, M.B. van der Mark, C.W.J. Beenakker, Nature 375, 767 (1995).

\section{The Simple Adiabatic Theory}

The main theoretical ideas about one-dimensional conduction have been around since the $1950^{6}$, but nobody actually realised their implication for the physics of breaking an electrical contact. Consider two wide electron gas reservoirs connected by a narrow one-dimensional conductor (see figure 3 ). We assume that the electrons move ballistically between the two reservoirs - that is, the electrons are only scattered by the boundaries of the constriction. The electrons in the nanowire then behave like a free $1-\mathrm{D}$ electron gas along $(\mathrm{Oz})$. Because of the small size $a$ of the constriction, there is a large confinement energy in the transverse directions $(\mathrm{x}, \mathrm{y})$ leading to a large splitting of the electronic levels. The energy of the electrons in the nanowire can be written as:

$$
E_{n}(k)=\left(n_{x}^{2}+n_{y}^{2}\right){\frac{\hbar^{2} \pi^{2}}{2 m a^{2}}(\text { quantized })}+\frac{\hbar^{2}}{2 m} k_{z \text { (contimuous })}^{2}
$$

which is the sum of a quantised contribution (transverse motion) and a continuous contribution (free diffusion along Oz); $n_{x}$ and $n_{y}$ are indices for the possible transverse modes. The corresponding band structure of a more realistic nanowire is shown in figure $2 a$. A difference of chemical potential $\left(\mu_{1}-\mu_{2}\right)$ is obtained by applying a small potential difference, $\mathrm{V}_{\text {bias }}=\left(\mu_{1}-\mu_{2}\right) / e$, between the two electron reservoirs. Then the number of electrons per mode $n$ contributing to the current is $1 / 2 g_{n}\left(E_{f}\right)\left(\mu_{1}\right.$ $\left.\mu_{2}\right)$, where $g_{n}\left(E_{f}\right)$ is the density of states at the Fermi level of mode $n$. The charge current in mode $n$ is then equal to:

$$
I=e v_{F n} \frac{g_{n}\left(E_{f}\right)}{2}\left(\mu_{1}-\mu_{2}\right)
$$

where $v_{F n}$ is the group velocity at the Fermi level of mode $n$. The density of state (DOS) of a 1-D electron gas at the Fermi level is given by $g_{n}\left(E_{f}\right)=1 / h v_{F n}$. The conductance of mode $n$ is then equal to:

$$
G_{0}=\frac{I}{V_{\text {bias }}}=\frac{2 e^{2}}{h}
$$

Remarkably each occupied sub-band contributes the same current (figure $2 b$ ), since the $1 / v_{F}$ dependence of the 1-D DOS cancels with the group velocity. Herein is the origin of the conductance quantisation. If we have $N$ channels contributing, and each channel has a transmission $T_{i}$, then the total conductance is given by the Landauer formula:

$G=\frac{2 e^{2}}{h} \sum_{i=1}^{N} T_{i}$

So, what happens when an electrical contact is broken? At first we have a wide contact with an effectively infinite number of modes contributing to the conductance. As the contact opens, the size of the metallic constriction connecting the two electrodes shrinks. Thus the spacing between the energy levels increases (figure $2 b$ ), and as the modes rise above the Fermi level one after the other, the conductance drops in steps of $G_{0}$. For very narrow constrictions $(\mathrm{a} \sim 5 \AA)$, the energy splitting between the different modes is of the order of $1 \mathrm{eV}$. So it is possible to observe these quantum confinement effects even at room temperature. Surprisingly, the experiments indicates that the transmission coefficients $T_{i}$ in the Landauer formula are very close to 1 (or o). Previously these coefficients were expected to range randomly between 0 and 1 . 